\title{
A39 TOLL-LIKE RECEPTOR-4 SIGNALLING IS SPECIFICALLY TAK1-INDEPENDENT IN SYNOVIAL FIBROBLASTS
}

Ben T van den Brand, ${ }^{1}$ Shahla Abdollahi-Roodsaz, ${ }^{1}$ Onno J Arntz, ${ }^{1}$ Michael Kracht, ${ }^{2}$ Jeroen Geurts, ${ }^{1}$ Wim B van den Berg, ${ }^{1}$ Fons A J van de $L 00^{1}{ }^{1}$ Department of Rheumatology, Radboud University Nijmegen Medical Centre, Nijmegen, The Netherlands; 2University Giessen, Rudolf-Buchheim-Institute of Pharmacology, Frankfurter Strasse 107, 35392 Giessen, Germany

\subsection{6/ard.2010.148965.9}

Background and objective Activated synovial fibroblasts are key players in the pathogenesis of rheumatoid arthritis (RA) by driving inflammation and joint destruction. Numerous molecules including cytokines and toll-like receptor (TLR) ligands induce pro-inflammatory signalling and gene expression through a hierarchical network of kinases. Upstream mitogen-activated protein kinase kinase kinases (MAP3Ks) represent an attractive target for RA treatment. In this study the authors sought to determine the role of the MAP3K transforming growth factor $\beta$ activated kinase1 (TAK1) in cytokine and TLR-mediated signalling.

Materials and methods TAK1 activity was inhibited using either a small molecule inhibitor 5Z-7-oxozeaenol or lentivirally overexpressed kinase-inactive TAK1-K63W mutant in murine embryonic and human dermal and synovial fibroblasts. Fibroblasts were stimulated with interleukin 1 (IL-1), tumour necrosis factor (TNF), TLR2 or TLR4 agonists and responses were evaluated using transcriptional reporters and analysis of gene expression of collagenases (matrix metalloproteinases (MMP3, MMP13)), cytokines (IL-1 $\beta$, IL- 6 ) and chemokines (IL-8, MCP-1).

Results TAK1 inhibition abrogated cytokine- and TLR-induced activation of nuclear factor $\kappa$-light-chain-enhancer of activated $B$ cells (NF-KB) and Saa3-promoter reporters in mouse and human 
dermal fibroblasts. In synovial fibroblasts, TAK1 crucially regulated IL-1 and TNF mediated NF-אB, but not Saa3-promoter activation. Furthermore, TAK1 was required for inducible mRNA expression of IL-1 $\beta$, IL-6, IL-8, MMP3 and MMP13, but not MCP-1, in response to IL-1, TNF and TLR2 agonist. Unexpectedly, TLR4-induced NF- $\mathrm{BB}$ activation and gene expression was fully TAK1-independent as found using the TAK1 inhibitor or kinase inactive mutant. Western blots demonstrated IL-1-inducible p38 and p65 phosphorylation and inhibition by the TAK1 inhibitor 5Z-7-oxozeaenol, whereas no effect of TAK1 inhibitor on lipopolysaccharides signalling in RA-SF.

Conclusions In general, TAK1 plays a prominent role in regulation of IL-1- and TNF mediated signalling in fibroblasts. Interestingly, TLR4 signalling is specifically TAK1-independent in synovial fibroblasts. Consequently, therapeutic TAK1 inhibition in arthropaties may not dampen the damage-associated molecular pattern-mediated TLR4 activation of synovial fibroblasts. 